Form fact or i $n$ persi stent hol e burning i $n$ mul ti pl e scattering opt i cal medi a

\begin{tabular}{|l|l|}
\hline 著者 & Tomit a Nakot o, Tat suno Shuho \\
\hline $\begin{array}{l}\text { j our nal or } \\
\text { publ i cat i on t i t l e }\end{array}$ & Physi cal Revi ew E \\
\hline vol une & 73 \\
\hline number & 6 \\
\hline page range & $066622-066622$ \\
\hline year & 2006-06 \\
\hline 出版者 & Aner i can Physi cal Soci et y \\
\hline 権利 & (c) 2006 The Aner i can Physi cal Soci et y \\
\hline URL & ht t p: //hdl . handl e. net /10297/598 \\
\hline
\end{tabular}




\title{
Form factor in persistent hole burning in multiple scattering optical media
}

\author{
Makoto Tomita and Shuho Tatsuno \\ Department of Physics, Faculty of Science, Shizuoka University, 836, Ohya, Suruga-ku Shizuoka, 422-8529, Japan
}

(Received 24 April 2006; published 23 June 2006)

\begin{abstract}
This paper presents detailed experiments on persistent hole burning in photoreactive and multiple scattering media. The dependence of the hole shape and width on the sample thickness, transport mean free path, and absorption length was measured. The experimental results show good agreement with a previously reported theoretical calculation on the basis of a diffusion approximation [Phys. Rev. E 70, 046606 (2004)].
\end{abstract}

DOI: 10.1103/PhysRevE.73.066622

PACS number(s): 42.25.Dd, 42.30.Ms, 42.70.Mp

The light injected into a multiple scattering medium travels a fairly long path length, undergoing multiple scattering [1]. In general, interference effects produced by light that traveled a long optical path would be highly sensitive to the incident beam conditions. For this reason, the multiple scattering medium has potentially a high ability to select frequencies or wave vectors. This property has been wildely utilized in optical measurements and applications [2]. Sharp spectral structures in a random laser [3-5] and high tunability of a speckle mirror [6] can also be attributed to this selectivity of the multiple scattering medium.

Hole burning in a multiple scattering medium may be another example that uses this property of the medium. In hole burning, first, a strong writing beam with the incident frequency $\omega_{W}$ and wave vector $k_{W}$ is injected into the multiple scattering medium. The speckle pattern produced by the writing beam is recorded inside the medium through a photoreactive process. After the pattern is recorded, the luminescence intensity from the sample excited by a reading beam with frequency $\omega_{R}$ and wave vector $k_{R}$ is measured as a function of $\Delta \omega=\omega_{W}-\omega_{R}$ or $\Delta k=k_{W}-k_{R}$. When the frequency and the wave vector of the writing and reading beams are the same, the spatial fluctuation patterns produced by the two beams coincide with each other. The reading beam, then, excites only the photobleached parts of the medium, where the luminescence intensity is reduced. We observe a hole in the luminescence intensity as a function of $\Delta \omega$ or $\Delta k$ [7-10]. Although this phenomenon has great potential in a wide range of photonic applications, the performance of hole burning is complicated and depends on various characteristics of the medium. Here, we present an experimental study of the form factor in persistent hole burning in a multiple scattering medium. Specifically, we experimentally examined the dependence of hole shape and width on the sample thickness $L$, transport mean free path $l^{*}$, and absorption length $l_{a}$ in the $\Delta k$ domain to map the contributions to the hole from different paths.

In all samples, a spiropyran derivative photochromic dye $\quad 1^{\prime}, 3^{\prime}$-dihydro-1', $2^{\prime}, 3^{\prime}$-trimethyl-6-nitrospiro[2H-1benzopyran-2, $2^{\prime}-(2 \mathrm{H})$ indole] (SP1) was used as the photoreactive material. After ultraviolet (uv) light irradiation, the sample shows an absorption band at $570 \mathrm{~nm}$ (colored state). When visible laser light is used for irradiation, the luminescence intensity at $660 \mathrm{~nm}$ from the colored state decreases, accompanied by the disappearance of the $570 \mathrm{~nm}$ absorption band (colorless state). We prepared an SP1-doped thin polymethyl methacrylate (PMMA) film and ground it into a mi- cro powder. The powder was compacted between two glass plates and used as the samples. The transport mean free path was determined from the total transmission measurement, while the absorption length was calculated from the cross section and the concentration of the photochromic dye. We employed a sample of $L=1.0 \mathrm{~mm}, l^{*}=60 \mu \mathrm{m}$, $l_{a}=28800 \mu \mathrm{m}$; hence the diffusive absorption length $L_{a}=\sqrt{l^{*} l_{a} / 3}=760 \mu \mathrm{m}$ for the standard sample, and the sample parameters were varied around these values. In the standard sample, the condition $l^{*}<L$ is satisfied; therefore we can analyze the hole on the basis of a diffusion approximation. We can experimentally examine the confined geometry effect, varying the sample thickness from 0.2 to $1.0 \mathrm{~mm}$. To study the effect of absorption on the hole shape, we controlled the concentration of the photochromic dye in the PMMA powder, while to examine the effect of the transport mean free path, we mixed $\mathrm{TiO}_{2}$ microparticles of $0.25 \mu \mathrm{m}$ average diameter into the PMMA powder.

The setup for the hole burning experiments was similar to that used in a previous report [9]. We used an $\mathrm{Ar}^{+}$laser of $514.5 \mathrm{~nm}$ for the writing and reading beams. The sample was set on a stage and rotated by a stepping motor and the incident angle $\theta$ between the beam and the sample was changed. Before the hole burning experiments, samples were exposed to uv light and prepared in the initial colored state. First, the

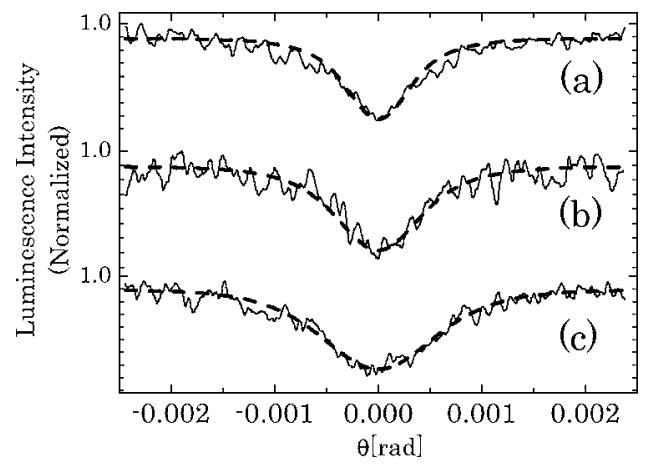

FIG. 1. Solid lines are experimental results for the persistent hole as a function of angular shift $\theta$ between the writing and reading beams. The sample thickness is $L=$ (a) 1.0 , (b) 0.4 , and (c) $0.2 \mathrm{~mm}$. The transport mean free path and the absorption length are $l^{*}=60 \mu \mathrm{m}$ and $l_{a}=28800 \mu \mathrm{m}$, respectively. Dashed lines are theoretical curves calculated on the basis of Eq. (1), where a Gaussian shaped function is convoluted as the experimental angular resolution. 


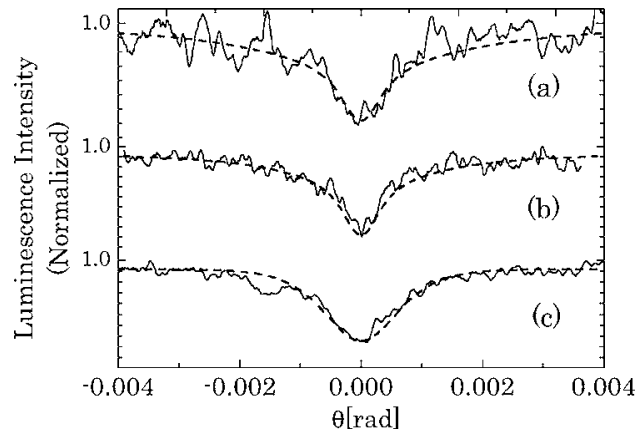

FIG. 2. Solid lines are experimental results for the persistent hole as a function of angular shift $\theta$. The transport mean free path and the absorption length are (a) $l^{*}=11 \mu \mathrm{m}, l_{a}=28800 \mu \mathrm{m}$, (b) $l^{*}=17 \mu \mathrm{m}, l_{a}=28800 \mu \mathrm{m}$, and (c) $l^{*}=60 \mu \mathrm{m}, l_{a}=580 \mu \mathrm{m}$. The sample thickness is $L=1.0 \mathrm{~mm}$ in all samples. Dashed lines are theoretical curves calculated on the basis of Eq. (1), where the experimental angular resolution is convoluted. Note that the horizontal scale is reduced; therefore the hole width is broad, compared with Fig. 1.

laser beam was irradiated onto the sample and the volume speckle pattern was recorded through the photochromic process. Then, the beam intensity was attenuated by a factor of 100 and used as the reading beam. The luminescence excited by the reading beam was collected in a reflection geometry and measured as a function of the angle of the rotational stage. The experimental angular resolution was $0.6 \mathrm{mrad}$,
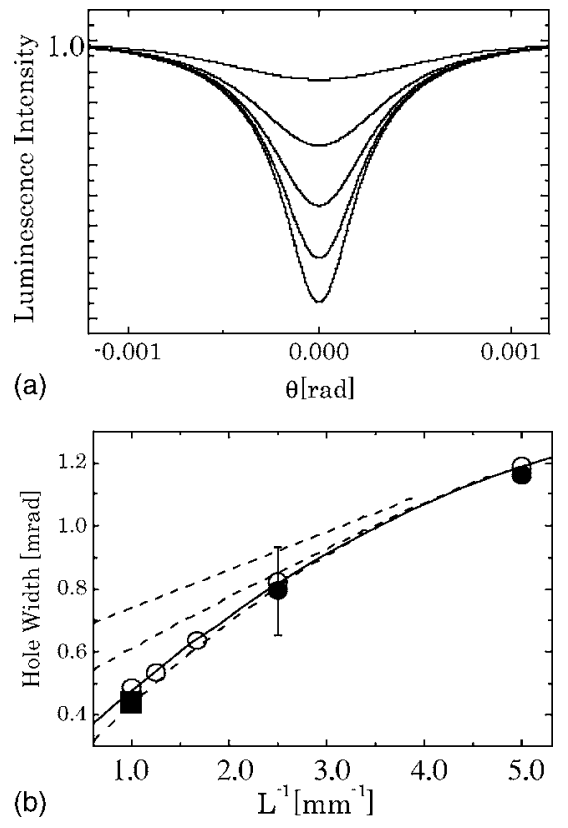

FIG. 3. (a) Calculated hole shapes for different sample thickness. From top to bottom, $L=0.2,0.4,0.6,0.8$, and $1.0 \mathrm{~mm}$, where $l^{*}=60 \mu \mathrm{m}$ and $l_{a}=28800 \mu \mathrm{m}$. (b) Hole width as a function of the inverse of the sample thickness. The solid line is the calculated curve for $l_{a}=28800 \mu \mathrm{m}$, while the three dashed curves are $l_{a}=4000$ and $8000 \mu \mathrm{m}$ and infinity, respectively. The transport mean free path is $l^{*}=60 \mu \mathrm{m}$. The solid symbols are experimental results shown in Fig. 1. The solid square is for the standard sample. The open circles correspond to the simulation shown in Fig. 3(a).
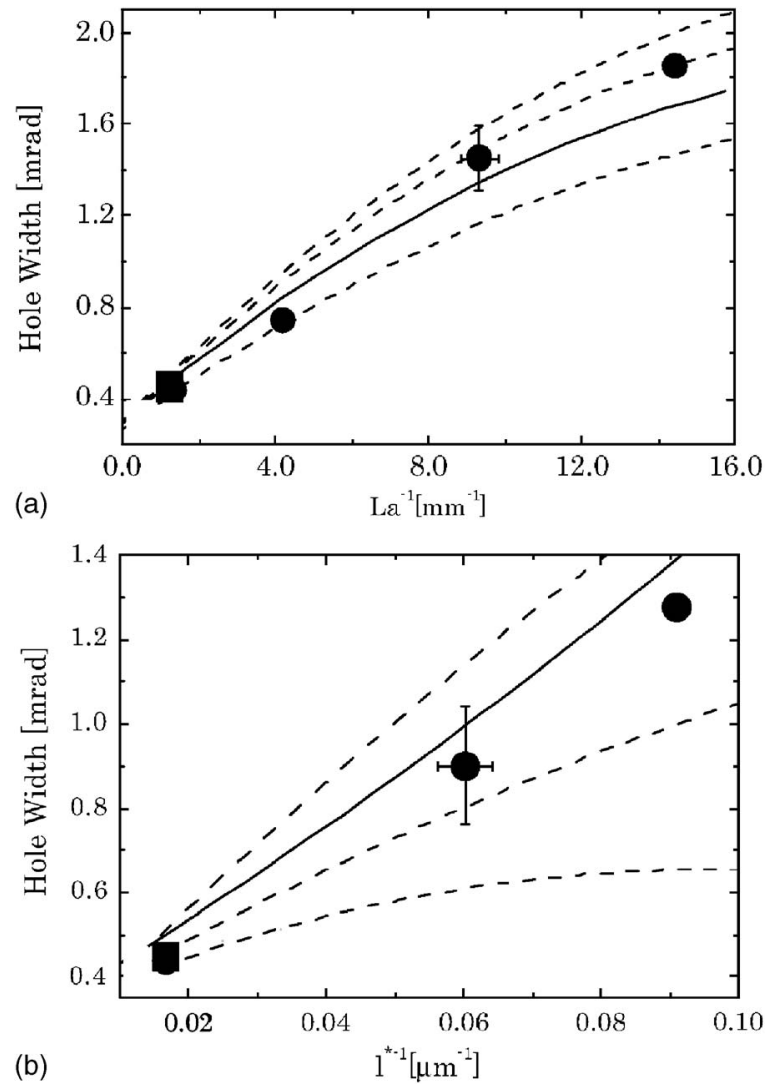

FIG. 4. (a) Hole width as a function of the inverse of the diffusive absorption length. The solid line is the calculated curve for $l^{*}=60 \mu \mathrm{m}$. The dashed curves are, from top to bottom, $l^{*}=45,50$, and $80 \mu \mathrm{m}$, respectively. The sample thickness is $L=1.0 \mathrm{~mm}$. The solid circles are experimental results including the data shown in Fig. 2(c). The solid square is for the standard sample. (b) Hole width as a function of the inverse of the transport mean free path. The solid line is calculated curve for $l_{a}=28800 \mu \mathrm{m}$. The dashed curves are, from top to bottom, $l_{a}=20000$ and $60000 \mu \mathrm{m}$ and infinity, respectively. $L=1.0 \mathrm{~mm}$. The solid square is for the standard sample. The solid circles are experimental results including the data shown in Figs. 2(a) and 2(b).

which arose mainly from the beam divergence of the $\mathrm{Ar}^{+}$ laser used in the experiments. The photochromic reaction from the colored to the colorless state and the reverse process were stable and repeatable.

The hole shows a characteristic time development, reflecting the statistics of the intensity fluctuations in the speckle inside the medium [9]. In the investigation of the hole shape, it is very important to take this time development effect into account. In the previous paper, we distinguished three types of samples according to the photoreactive processes. In the type II sample in the notation used in Ref. [9], we consider a photoreaction in which the absorption is unchanged, while the luminescence intensity decreases. On the other hand, in the type III sample, we consider a photoreaction in which the absorption spectrum or the coefficient changes under the laser irradiation. The photochromic dye used in the present experiments is classified as a type III sample. At the initial stage of the time development, the hole can show shape and width intrinsic to the sample, which is determined by the 
wave propagation in the multiple scattering medium. On the other hand, at a later stage, the hole shape is determined by the saturation effect in the writing process. In order to examine the dependence of the hole on the sample parameters, we should observe the hole at the initial stage $t \eta_{a} \eta_{p} I_{W} \equiv \kappa I_{W} \ll 1$, where $t$ is the writing time, $I_{W}$ is the intensity of the writing beam inside the medium, $\eta_{a}$ is the absorption cross section, and $\eta_{p}$ is the efficiency of the photoreaction [9]. From the point of view of experiments, however, shallow holes at the initial stage are disadvantageous to the signal-to-noise ratio. In the experiments reported here, this tradeoff is taken into account and the hole shape and width are analyzed at the initial stage of the hole burning with a hole depth of $0.05-0.1$ of the initial luminescence intensity. In the present paper, we do not discuss the type I sample in detail, because the photoreactive material is placed outside the medium in this sample, and the hole does not show the characteristic behavior relevant to the fluctuations generated inside the multiple scattering medium.

Figure 1 shows experimental observation of the hole as a function of $\theta$ for different sample thicknesses. The transport mean free path and the absorption length are $l^{*}=60 \mu \mathrm{m}$ and $l_{a}=28800 \mu \mathrm{m}$, respectively. In the luminescence intensity, a dip as a function of $\theta$ is clearly seen; hence, we can observe a hole in the photoreactive and multiple scattering medium. The sample thickness is varied from $L=0.2$ to $1.0 \mathrm{~mm}$, ranging from the $L_{a}<L$ to the $L_{a}>L$ region. As the sample thickness is decreased, the hole width increases. It is also seen that the bottom of the hole in the thin sample [Fig. 1(c)] is more rounded compared with the thick sample [Fig. 1(a)]. Figure 2 shows the experimental observation of the hole as a function of $\theta$ for different transport mean free paths $l^{*}$ and absorption lengths $l_{a}$. Parameters are given in the figure caption. The hole width is summarized in Fig. 3(b) as a function of the inverse of the sample thickness $L^{-1}$, and in Figs. 4(a) and $4(\mathrm{~b})$, as a function of the inverse of the diffusive absorption length $L_{a}^{-1}$ and the transport mean free path $l^{*-1}$, respectively. In our measurements, the experimental angular resolution is comparable to the minimum hole width observed; therefore it is necessary to deal with the experimental resolution correctly. In the plots of the hole width, a Gaussian shaped function of 0.6 mrad was deconvoluted from the experimental data as the experimental angular resolution. As is seen in the results shown in Figs. 1 and 2, the effects of both finite thickness and absorption on the hole width are similar. These effects are readily explained as the effect of removal of the contribution of the longer path in the writing process to the hole formation [8-10]. Since the speckle pattern produced by light traveling along the long trajectories is more sensitive to the changes in the frequency or wave vector, the removal of these trajectories results in wide holes.

While the observed dependence of the hole shape and width can readily be interpreted by the removal of the longer trajectories in the medium, a quantitative analysis is also possible on the basis of the diffusion approximation. For the slab geometry, the dependence of the hole at the initial stage of the time development is approximated as [9]

$$
\begin{aligned}
H^{R}(\Delta k, \Delta \omega)= & \int_{l^{*}}^{L} d z \frac{\sinh \left[(L+C-z) \alpha_{l}\right]}{\sinh \left[(L+2 C) \alpha_{l}\right]}\left(\frac{\sinh [(L+C-z) \alpha]}{\left\{\sinh [(L+2 C) \alpha]+\kappa I_{W} \sinh [(L+C-z) \alpha]\right\}}\right. \\
& -\kappa I_{W} \frac{\sin h^{2}[(L+2 C) \alpha]}{\left\{\sinh [(L+2 C) \alpha]+\kappa I_{W} \sinh [(L+C-z) \alpha]\right\}^{2}} \\
& \left.\times\left|\frac{\sinh [(L+C-z) \beta]}{\sinh [(L+2 C) \beta]}\right|^{2}\right) / \int_{l^{*}}^{L} d z \frac{\sinh [(L+C-z) \alpha] \sinh \left[(z+C) \alpha_{l}\right]}{\sinh [(L+2 C) \alpha] \sinh \left[(L+2 C) \alpha_{l}\right]}
\end{aligned}
$$

for the reflection geometry, where $\beta=\gamma+i \delta$ $=\sqrt{\Delta k^{2}+\left(1 / \tau_{a}+i \Delta \omega\right) / D}, \tau_{a}=l_{a} / c, c$ is the velocity of light inside the medium, $D$ is the diffusion constant, and $\alpha$ and $\alpha_{l}$ are the absorption coefficients for the writing and reading beams and for the luminescence light, respectively. The $z$ axis is taken to be perpendicular to the incident surface, and we set the absorbing wall at $z=-C$ and $L+C$, where $C=\left(2 l^{*} / 3\right)(1+\rho) /(1-\rho)$ is the extrapolation length and $\rho$ is the internal reflection coefficient. Figure 3(a) shows the theoretically calculated hole shape on the basis of Eq. (1) for different sample thicknesses, where the background level of the luminescence intensity has been scaled so that all curves have the same intensity. When the sample thickness is decreased, the luminescence from deeper regions of the sample is removed. This effect makes the absolute hole depth shal- low and the bottom of the hole more rounded. In Figs. 1 and 2 , we also plotted the calculated hole shape with dashed lines, where a Gaussian shaped function is convoluted over the calculated curves based on Eq. (1) as the experimental resolution. A similar calculation was performed for samples of different transport mean free paths $l^{*}$ and absorption lengths $l_{a}$, and the results are summarized in Figs. 3 and 4. In Fig. 3, the hole width decreases as the sample thickness is increased. The hole width asymptotically approaches the value determined by the diffusive absorption length $L_{a}$, as the sample thickness is increased as $L^{-1} \rightarrow 0$. In Fig. 4(a), the hole width decreases when $L_{a}$ is increased. When the sample thinness is finite, the hole width asymptotically approaches the value determined by the sample thickness as the diffusive absorption length is increased as $L_{a}^{-1} \rightarrow 0$. In the fitting pro- 
cess, when the condition $l^{*} \ll L$ is not satisfied, we found that the actual calculated hole shape, specifically in the hole tail regions, is very sensitive to the lower boundary of the integration in Eq. (1), which was set as $l^{*}$. Since a welldeveloped speckle exists in the region of $l^{*}<z$, this is reasonable. We can find a set of parameters, transport mean free path $l^{*}$, and absorption length $l_{a}$ for the series of experiments; therefore it is concluded that the experiments recapture the theory.

The effects of finite thickness and absorption on the line shape of the coherent backscattering peak from a disordered medium have also been investigated [11]. The coherent backscattering peak arises from a constructive interference between two amplitudes of a coherent wave traveling the same path in opposite directions, and is the manifestation of the weak localization of light $[1,12,13]$. By the termination of the coherent random walk of photons through confined geometry or absorption effects, the contribution of different time-reversed paths to the line shape has been determined. The most important parameter to determine the line shape of the coherent backscattering peak is the transport mean free path. On the other hand, in the present hole burning experi- ment, the sample thickness is a crucial parameter. This difference appears most clearly in the limit that the absorption becomes zero. The line shape of the hole in the $\Delta k$ domain in the reflection geometry does not depend on the transport mean free path as long as $l^{*} \ll L$, while that of the coherent backscattering peak does. This effect is seen in the bottom dashed curve in Fig. 4(b), where the hole width approaches a constant value when the transport mean free path is decreased as $l^{*} \ll L$. This difference arises because the coherent backscattering peak is composed directly by the diffusively reflected flux, while in hole burning the luminescence excited by the diffused intensity of the writing beam inside the medium is observed.

In summary, we experimentally studied persistent hole burning in photoreactive and multiple scattering media. The shape and width depend on the various sample parameters, such as the sample thickness, transport mean free path, and absorption length. The experimental results show good accordance with the previously reported theoretical calculation. In addition to the fundamental interest in wave propagation in multiple scattering medium, this phenomenon is potentially useful in photonic applications.
[1] Scattering and Localization of Classical Waves in Random Media, edited by P. Sheng (World Scientific, Singapore, 1990).

[2] J. C. Dainty, Laser Speckle and Related Phenomena (SpringerVerlag, Berlin, 1984).

[3] H. Cao, Y. G. Zhao, S. T. Ho, E. W. Seelig, Q. H. Wang, and R. P. H. Chang, Phys. Rev. Lett. 82, 2278 (1999).

[4] H. Cao, J. Y. Xu, D. Z. Zhang, S.-H. Chang, S. T. Ho, E. W. Seelig, X. Liu, and R. P. H. Chang, Phys. Rev. Lett. 84, 5584 (2000).

[5] Sushil Mujumdar, Marilena Ricci, Renato Torre, and Diederik S. Wiersma, Phys. Rev. Lett. 93, 053903 (2004).

[6] Paulo C. de Oliveira, John A. McGreevy, and N. M. Lawandy, Opt. Lett. 22, 700 (1997).
[7] A. Kurita, Y. Kanematsu, M. Watanabe, K. Hirata, and T. Kushida, Phys. Rev. Lett. 83, 1582 (1999).

[8] M. Tomita, T. Ito, and S. Hattori, Phys. Rev. B 64, 180202(R) (2001).

[9] Makoto Tomita, Kentarou Ono, and Shuho Tatsuno, Phys. Rev. E 70, 046606 (2004).

[10] T. Ito and M. Tomita, Phys. Rev. E 69, 036610 (2004).

[11] S. Etemad, R. Thompson, M. J. Andrejco, Sajeev John, and F. C. MacKintosh, Phys. Rev. Lett. 59, 1420 (1987).

[12] A. A. Chabanov, M. Stoytchev, and A. Z. Genack, Nature (London) 404, 850 (2000).

[13] Valery Milner and Azriel Z. Genack, Phys. Rev. Lett. 94, 073901 (2005) 